\title{
Ischaemic Bowel Disease : A Case Report
}

\author{
Maj KJ Singh", Maj Rajiv Gupta ${ }^{+}$, Lt Col Vk Popli ${ }^{\#}$
}

MJAFI 2002; $58: 265-266$

Key Words : Haemorrhagic gastroenteropathy; Ischaemic colitis.

\section{Introduction}

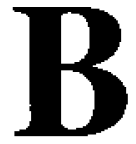
owel infarction is an uncommon but grave disorder that imposes a 50 to $75 \%$ mortality rate [1]. The small intestine alone, the colon alone or sometimes both may sustain hypoxic injury related to various causes of vascular compromise.

\section{Case Report}

A 26 year old serving soldier, a non smoker and non drinker, presented on 27 Mar 2000 with pain abdomen of 6 days duration. The pain started on 21 March 2000 after lunch. It started in right lower abdomen, mild to moderate in intensity, continuous, non-radiating and with no specific aggravating or relieving factors. The patient had one vomiting, non-bilious, non-projectile and no haematemesis. He also had loose motions 4-5, some of them mixed with attered blood. On 22 and 26 March 2000 he took some medication without any relief. The pain increased in intensity on 26 March 2000 and spread to the entire abdomen. It also started radiating to the right shoulder. Interestingly he had relief on sitting up and pain used to aggravate on lying down unlike the pain in peritonitis. He had not passed flatus or stool for the last 24 hours. Since he was on a snow bound post, he could not be evacuated immediately to the Field Ambulance and could reach only on 27 March 2000.

Examination revealed that the patient was in pain, looking ill and his tongue was dry and coated. He was febrile with a temperature of $100^{\circ} \mathrm{F}$, pulse $118 / \mathrm{min}$ and BP $110 / 70$ $\mathrm{mm} \mathrm{Hg}$. There was mild pallor, no icterus or lymphadenopathy. His abdomen was mildly distended with guarding all over, maximum in the right jliac fossa and suprapubic region. Shifting dullness was present and bowel sounds were sluggish. Genitals and hernial sites were normal. Perrectal examination revealed tenderness all around. There was no stool or blood on the finger stall. Limited investigations were available at the centre. Haemogram was within normal limits. $\mathrm{X}$-ray abdomen erect view showed multiple fluid levels with a mild dilatation of the intestinal loops. The patient was accepted in ASA IV E for an emergency exploratory laparotomy.

At surgery there was haemoperitoneum with one litre of altered blood and a mild foul smell. No pus or food residue was found. After aspiration and mopping, a mass was felt at the lower end of the sigmoid colon going on to the rectosigmoid junction. To this a loop of small intestine was adherent. The small intestine was thick, edematous, rubbery and the mesentery was thickened. On separating the loops carefully, a $15 \times 10 \mathrm{~cm}$ cauliflower like growth was found at the lower sigmoidal junction, and a loop of small intestine adherent to it seemed to have been invaded by it. The mass was congested and dusky. The serosa was tom at places and it seemed that the colon had perforated. It was carefully assessed and on table appeared like a carcinoma. Hence, a resection with $5 \mathrm{~cm}$ margin on either side was done and a Hartmann's procedure was performed. The part of small intestine which seemed invaded was resected and anastomosed. Proper lymph node clearance could not be done. Post operative recovery was uneventful, colostomy moved on the $2^{\text {nd }}$ postoperative day and the patient was transferred to Command Hospital (Westem Command) on the $6^{\text {th }}$ post operative day. He then underwent closure of colostomy.

Histopathology of both the large and small intestine reported that the lumen had clotted blood with necrotic cellular debris. Lamina propria was edematous and had inflammatory infiltrates. There was intense congestion of the blood vessels. Areas of lining mucosa showed denudation and ulceration at some places while being well delineated at others. An opinion of Ischaemic Intestinal Disorder. "Haemorhagic Gastro-enteropathy" was given.

\section{Discussion}

The small intestine alone, the colon alone or sometimes both may sustain hypoxic injury related to various causes of vascular compromise. Depending on the severity in reduction of blood flow, three morphological patterns are arbitrarily described by Morson as follows :

1. Infarction or gangrene of the bowel, i.e. transmural ischaemia; necrosis due to the absence or a marked reduction in the blood perfusion.

2. Haemorthagic gastroenteropathy or Transient Ischaemia : characterized by haemorrhage and necrosis limited to the mucosa and submucosa with sparing usually of the deeper layers, related to less extreme reduction in the blood flow.

3. Chronic ischaemia or Ischaemic stricture : leading in time to fibrotic narrowing of the affected bowel.

Haemorthagic gastroenteropathy may involve any level of the gut from stomach to the anus. It is a segmental disease, the splenic flexure being the classic

\footnotetext{
"Graded Specialist(Surgery)", "Graded Specialist (Anaesthesiology), "Commanding Officer, 328 Field Ambulance. C/o 56 APO.
} 
site of involvement due to relative paucity of blood supply, however, other sites including the rectum can be involved. Spontaneous non occlusive ischaemic colitis involving only the right colon is infrequent occurrence. The correct diagnosis is usually not considered in the case [2]. It appears to relate to hypoperfusion damaging only the inner layers of the gut while sparing the outer layers of muscularis and serosa. There is a reduction in the effective circulating blood volume with shunting of blood to the vital organs and away from the splanchnic bed. The lesions may be multifocal or continuous and widely distributed throughout the gastrointestinal tract.

Affected areas of the bowel may appear dark red or purple owing to accumulated luminal haemorrhage regardless of whether the arterial or venous side is involved. In the early stages, the segment of bowel appears intensely congested and dusky to purple red with small to large foci of subserosal and submucosal ecchymotic discolouration. Later, the wall becomes edematous, rubbery, thickened and haemorthagic, commonly at this stage and the lumen contains sanguinous mucus or frank blood. In arterial occlusion, the demarcation is usually sharply defined but in venous occlusions the area of dusky cyanosis fades gradually into the adjacent segments of normal bowel. The pathogenesis involves nonocclusive hypoperfusion of the intestinal tract. Why the resultant lesions are patchy, remains unexplained. The presumed aetiologies are numerous; however it typically may occur spontaneously in the absence of major vascular occlusion and in the presence of normal intestine elsewhere [3].

Most cases occur in patients past 50 years of age and the usual associations are atherosclerosis, diabetes and status postvascular surgery. Shock and cardiac failure are important factors. Many patients have received digitalis and norepinephrine and some both. However, in this case the patient was very young and did not fit into any of the categories. Interestingly, it may relate to the spectrum and process of hypercoagulability and thrombosis seen in high altitude areas presenting variably as splenic infarcts, splenic and mesen- teric vein thrombosis, deep vein thrombosis, cerebrovascular accident in the young. The process manifests itself with sudden onset of bleeding and abdominal pain. Roentgenographic, endoscopic and intraoperative confusion with carcinoma may occur [4], as happened in this case. Another important differential diagnosis especially in the West is that of Crohn's disease. Although the latter can be seen in any age group, it should be remembered that ischaemic colitis is the most common form of colitis in the elderly.

Dilatation of the colon and physical signs of peritonitis are seen in severe cases. Abdominal frlms may reveal thumb printing from submucosal haemorrhage and edema. Barium enema is hazardous in the acute situation because of risk of perforation. Sigmoidoscopy or colonoscopy may detect ulcerations, friability or bulging folds from submucosal haemorrhage. Angiography is not helpful because a remediable occlusive lesion is rarely found.

Patients with mild abdominal pain, minimal leucocytosis and mild fever should be observed with anticipation of recovery in $2-3$ days time. If the ischaemia is more severe with marked abdominal pain and signs of fever and leucocytosis as was with our case, immediate laparotomy with resection and proximal colostomy should be resorted to. The morbidity and mortality are high among such patients [5].

\section{References}

1. Marshak RJ. Ischaemia of the colon. Mt Sinai J Med $1981: 48: 180-2$.

2. Schuler JG, Hudlin MM. Caecal necrosis :infrequent variant of ischaemic colitis. Report of five cases. Dis Colon Rectum 2000;43(\$):708-12.

3. Gandhi SK, Hanson MM, Vernava AM, Kaminski DL, Longo WE. Ischaemic Colitis. Dis Colon Rectum 1996;39(1):88-100.

4. Brandt LJ, Katz HJ, Wolf EL, Mitsudo S, Boley SJ. Simu]ation of colonic carcinoma by ischaemia. Gastroenterology 1985;88: I 137-42.

5. Kodner U, Fry RD, Fleshman JW. Bimbaum EH, Read TE. Colon, Rectum and Anus. In: Shwartz Principles and Practice of Surgery, $7^{\text {th }}$ ed $1999 ; 1284-5$. 